\title{
Interactive Scene Prediction for Automotive Applications
}

\author{
Andreas Lawitzky ${ }^{1}$, Daniel Althoff ${ }^{1}$, Christoph F. Passenberg ${ }^{1}$, Georg Tanzmeister ${ }^{1,3}$ \\ Dirk Wollherr ${ }^{1,2}$ and Martin Buss ${ }^{1}$
}

\begin{abstract}
In this work, a framework for motion prediction of vehicles and safety assessment of traffic scenes is presented. The developed framework can be used for driver assistant systems as well as for autonomous driving applications. In order to assess the safety of the future trajectories of the vehicle, these systems require a prediction of the future motion of all traffic participants. As the traffic participants have a mutual influence on each other, the interaction of them is explicitly considered in this framework, which is inspired by an optimization problem. Taking the mutual influence of traffic participants into account, this framework differs from the existing approaches which consider the interaction only insufficiently, suffering reliability in real traffic scenes. For motion prediction, the collision probability of a vehicle performing a certain maneuver, is computed. Based on the safety evaluation and the assumption that drivers avoid collisions, the prediction is realized. Simulation scenarios and real-world results show the functionality.
\end{abstract}

\section{INTRODUCTION}

Traffic reports [1] have shown that on average 93 people died each day on U.S. roads. However, this number has declined over the last two decades thanks to several safety systems in the vehicles. For a further decrease of the death toll, an important contribution is the development of additional driver assistance systems or even one step further completely autonomous driving systems. These systems shall help the driver of the vehicle to move the vehicle in a safe way, ideally also in a comfortable and fuel-conserving manner. Today, there are already lots of driver assistance systems integrated in vehicles which help the driver to avoid collisions or to save fuel such as the blind spot monitor or the intelligent shifters.

However, most assistant systems only consider the current state of the vehicle or the current traffic situation, but lack prediction of future situations. These systems are therefore hardly able to detect a dangerous situation at an early stage or to find the optimal gear with regard to the future motion. Thus, it is desirable to develop systems which are able to predict how surrounding traffic participants will behave.

This information could also be used by a driving assistant system, warning the driver about an upcoming dangerous situation, or for autonomous driving to generate safe and comfortable trajectories.

The goal of the approach presented in this work is to enhance the driving safety of vehicles in structured environ-

\footnotetext{
${ }^{1}$ Institute of Automatic Control Engineering and with the ${ }^{2}$ Institute for Advanced Study, Technische Universität München, 80290 München, Germany, ${ }^{3}$ BMW Group Research and Technology, 80992 München, Germany, $\{a . l a w i t z k y, d a, d w, m b\} @ t u m . d e$, cfpassenberg@lsr.ei.tum.de, georg.tanzmeister@bmw.de
}

ments. A more reliable prediction of future movements of vehicles shall be achieved by explicitly taking into account the mutual influence of traffic participants. We call this mutual influence interaction of the traffic participants. This interaction is important as explained with Fig. 1, which shows three vehicles on a highway. The red vehicle is overtaking the yellow vehicle at a high velocity, which in turns is approaching a much slower truck at the same time. Thus, the yellow vehicle has to either brake or change lanes in order to avoid a collision with the truck. With this knowledge, the driver of the red vehicle can adjust his prediction and react on the dangerous situation ahead of time. Therefore, a prediction without consideration of the interaction would be less reliable.

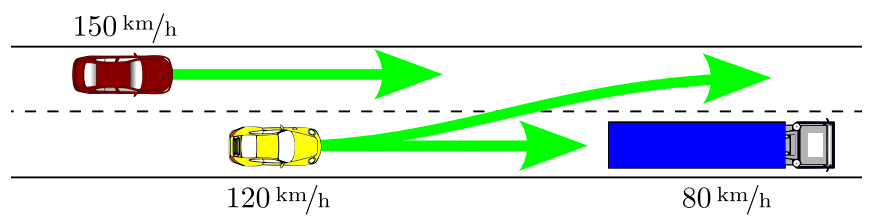

Fig. 1: Three vehicles are interacting in a highway scene.

In this work, a novel framework is presented which predicts the motion of vehicles on highways considering the interaction of the traffic participants, fulfilling the required time constraint for on-line computation. Although this work is explained with vehicles driving on highways, the approach can be used for all mobile robotic applications in dynamic and structured environments, where structured means that the motion space of the participants can be discretized without great losses.

\section{RELATED WORK}

In the following, different approaches for motion prediction and threat assessment are explained. In general these approaches can be grouped into learning based motion prediction, model based motion prediction, and motion prediction with a cognitive architecture. While learning based approaches for motion prediction learn from observation of the past movements of vehicles in order to predict the future motion, cognitive architectures try to reproduce human behavior and model based prediction use motion models. Former approaches ignore or insufficiently incorporate the influence of mutual interaction of the traffic participants.

The approaches in [2] and in [3], [4] aim at estimating the future position of a vehicle by its past movement. For that purpose, a database of motion primitives for different car actions is constructed preliminarily by recording trajectories. By observing the motion of a vehicle and the measurement of its current state, trajectories are assigned from the database 
to the car with a certain likelihood. Therefore, the former is using an extension of the Longest Common Subsequence (LCS), the Quaternion-based Rotationally Invariant LCS to compare the trajectories. The latter uses clustered trajectories in order to obtain typical motion patterns, where each cluster consists of several trajectories. These approaches allow one to estimate the future motion of a vehicle, assuming it follows the representative trajectory. Unfortunately, for good results the databases in these approaches have to contain an infeasible number of different trajectories in order to cover the amount of possible maneuvers. Additionally, the interaction of traffic participants is not considered as in following papers.

In contrast to the previously introduced approaches, the one presented in [5] and [6] is able to learn new motion patterns on-line while predicting the future movement of an object. For that purpose, the authors use Growing Hidden Markov Models. This approach worked well in their experiment on a parking lot.

In [7], a combined optimization of the motions of all vehicles in a particular traffic scene is performed using a cost function which punishes unsafe maneuvers of the vehicles. Additionally, this cost-function considers secondary goals like comfort and fuel consumption. However, this approach is based on the assumption that the traffic participants are centrally controllable.

In [8], the cognitive architecture Adaptive Control of Thought Rational (ACT-R) is introduced. The goal of this framework is to model human driving behavior including human perception, e.g. the steering motions are predicted in consideration of the delay, caused by cognition and human constraints regarding motor capabilities. The main focus of this framework is the comprehension of the way a vehicle is driven, but not to predict the actions of drivers, especially under mutual influence.

An approach using model based motion prediction for situation and threat assessment is presented in [9]. For that purpose, the authors introduce two parameters: Predicted Object Minimum Distance (PMD) and Predicted Time to Object Minimum Distance (TPMD), which serve as a measure for the threat of a situation, outperforming the commonly used Time to Collision parameter in their experimental results.

A framework for designing an accurate vehicle motion model is described in [10] and extended in [11]. This model includes the expected driver input, enabling an accurate long term prediction, using cost functions which depend on the individual driving style and intention of the driver. Information about the individual driving style is obtained by previous observation. The authors introduce an approach for the classification and recognition of maneuvers, which a driver likes to perform. The expected driver input can be computed by minimizing the cost function. It is possible to predict the future trajectory with respect to the postulated goals, the interaction of drivers is not considered.

In [12] an approach for vehicle maneuver identification and driver's intention prediction is presented. In order to recognize early which maneuver a driver is performing, a
Dempster-Schafer reasoning system is utilized, based on the theory of evidence. The authors use measurements like PMD, TPMD, Time to lane crossing (Tlc), or Distance from vehicle in path to identify the maneuvers. Although this approach helps to identify maneuvers the ego vehicle driver is likely to do, it does not estimate the future trajectories of the other vehicles, which makes it unsuitable for situation assessment.

In [13], the motion of a vehicle is described by a differential equation and abstracted by a Markov chain which consists of discrete states called reachable sets. They represent possible states which can be reached in a certain time interval. The transition probabilities of the Markov chain are obtained from the vehicle dynamics. The input is discretized and also abstracted by a Markov chain. From observation and heuristics the computation of the transition probabilities is achieved. In contrast to the former approaches, interaction of traffic participants is not neglected. For example, if a slower vehicle arises in the same lane, the probability of a lane change will increase. The safety assessment and motion prediction approach of [14] uses a dynamic motion model as well. With this model, the future trajectories can be computed for known control inputs. If this is available for all traffic participants, their trajectories can be checked for collisions. But as the inputs are unknown, the prior probability distribution has to be estimated with goal functions which model the drivers' behaviors. Monte Carlo sampling is used to find an approximate solution for several trajectories. Further [15] obtains a posterior distribution of the future inputs, assuming that drivers try to avoid collisions. With this posterior distribution, threat assessment can be done. However, constant velocities are assumed for the vehicles except the currently considered one. Thus, it is not considered that an obstacle may react on an upcoming vehicle.

An approach for motion prediction and risk assessment for lane-crossing scenes can be found in [16] and [17]. The authors separate the intention of drivers and the expectation of their motion. In order to avoid high complexity, Dynamic Bayesian Networks are used to model the motion of vehicles near intersections with the intention and expectation as hidden variables. Interference between these variables is assumed to be a measure for risk. Although this approach is tailor-made for crossings and uses a different theoretic background, it has parallels with the presented one as it considers the mutual influence of traffic participants. Likewise it distinguishes between the high-level intention of drivers and their situation-aware driving. This work also differs from works like [18], where the control input is implicitly chosen in a cooperative manner based on global knowledge of the desired goal.

In contrast to the presented algorithms, which do not consider the interaction of drivers sufficiently, the following novel algorithm focuses on the interaction of traffic participants in order to achieve more reliable motion prediction.

\section{PROBLEM STATEMENT}

This framework aims to predict the future motions of vehicles on highways. Like [15], this framework is based 
on the postulate that drivers will not perform maneuvers with high collision risks if safer options are possible. As shown before, the common approach to situation prediction implies independent prediction of each traffic participant. In contrast, the approach needs to be extended in order to respect the interaction of the drivers. Therefore, it is necessary to distinguish between the intention and the expected behavior of the drivers. The intention expresses which trajectory drivers want to drive having some high-level goal in mind, while the expectation incorporates knowledge about the current traffic scene. The first can be approximated with the presented common techniques, the latter is estimated with this novel approach. To achieve this, perfect perception of the traffic participants is assumed. With this, the framework will provide an improved estimation of the future motion, making use of the characteristics of structured environments like highways.

All traffic participants are able to perform different motion trajectories, superimposed in $m$. The future motion of the $i$-th vehicle can be modelled with a distribution function $f_{i}$, that assigns trajectories a certain probability. The desired output of the motion prediction is therefore the most accurate estimate of $f_{i}$.

\section{APPROACH}

The work assumes that the future motion of traffic participants can be estimated as a combination of the intention of each driver and the driver's local risk assessment to perform a maneuver. A situation based approach is presented in the following that unifies the intention and the threat estimate of all drivers, leading to an interaction-aware motion prediction.

Given a traffic scene, the probability that a collision will occur anywhere in the whole scene is

$$
P(C)=\int \operatorname{Ind}(C \mid m) f(m) \mathrm{d} m
$$

with $C$ the event of a collision. The function $f(m)$ combines the distribution functions $f_{i}$ to a continuous probability distribution for the infinite number of possible combinations of future motions of all vehicles in the road scene. These future trajectories determine how traffic situations will evolve in the future. The index-function $\operatorname{Ind}(C \mid m)$ equals one if at least one collision between two vehicles occurs, else zero.

With this information, this approach should improve the prediction of the situation. Fig. 2 illustrates that the possible trajectories a driver can perform is only restricted by the dynamic constraints. The number of possible trajectories is infinite even for a single vehicle. It is therefore not feasible to solve the combination of possible trajectories for all vehicles as in (1).

\section{A. Discretization of the Continuous Movement Space}

Making use of the fact that highways can be seen as a structured environment, the infinite number of possible movements a driver is able to perform can be approximated by a limited number of different maneuvers. Some examples for the discrete maneuvers on the highway include lane changes, acceleration, maintaining the speed, deceleration, and combinations as illustrated in Fig. 3 .

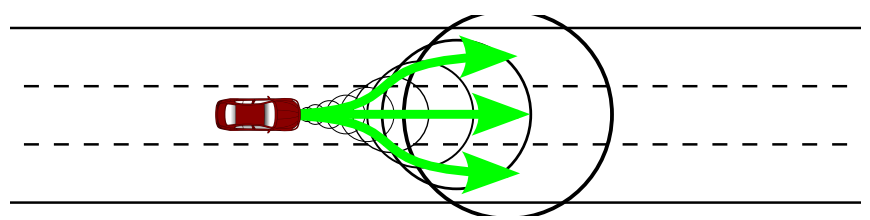

Fig. 2: The possible future positions of this vehicle on a highway are only restricted by the dynamic constraints. The black circles, called "Circles of Forces", define the area which a vehicle is able to reach in a particular time. The green arrows show possible future trajectories in this area.

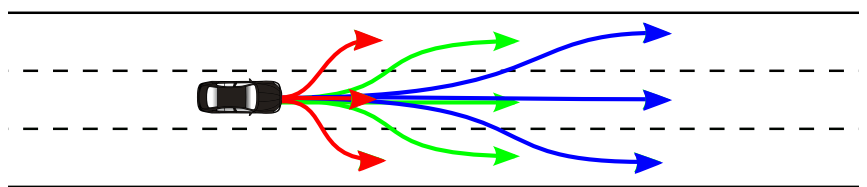

Fig. 3: The arrows show example trajectories for the allowed maneuvers. Red arrows label braking maneuvers. Green arrows show trajectories which keep their speed and the blue ones indicate an acceleration of the vehicle.

\section{B. Approximation of the Collision Probability}

For the approximation of (1) and simplification of the notation, some sets are introduced. The set $\mathcal{V}:=\left\{v_{1}, v_{2}, \ldots\right\}$ with $|\mathcal{V}|=v$ contains all $v$ traffic participants of a road scene. Each vehicle $v_{i}$ can perform maneuvers from the set $\mathcal{M}_{i}:=\left\{m_{i, 1}, m_{i, 2}, \ldots\right\}$ with $\left|\mathcal{M}_{i}\right|=m_{i}$. In order to obtain the possible future evolutions of a road scene, the sets $\mathcal{M}_{i}$ are permuted,

$$
\begin{gathered}
\mathcal{S}:=\mathcal{M}_{1} \times \mathcal{M}_{2} \times \cdots \times \mathcal{M}_{v}=\prod_{i=1}^{v} \mathcal{M}_{i}, \\
|\mathcal{S}|=\prod_{i=1}^{v} m_{i} .
\end{gathered}
$$

Hence, the set $\mathcal{S}$ consists of all $|\mathcal{S}|$ possible future evolutions of the current traffic scene i.e. all possible combinations of maneuvers. The set $\mathcal{S}$ contains all future scenes $s \in \mathcal{S}$, where $s$ is a $v$-tuple with $s=\left(s_{1}, s_{2}, \ldots, s_{v}\right)$. An example for a maneuver combination $s$ is illustrated in Fig. 4.

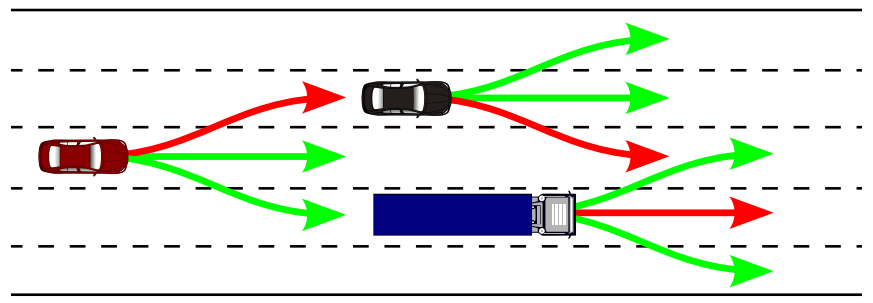

Fig. 4: The red arrows demonstrate one out of all possible maneuver combinations for this road scene. As each vehicle within this scene has three different opportunities to act, there are $m_{1} \cdot m_{2} \cdot m_{3}=27$ different maneuver combinations.

Each element $s_{i}$ of the tuple represents a certain maneuver $m_{i, j}$ of vehicle $v_{i}$, so $\mathcal{S}=\left\{\left(s_{1}, s_{2}, \ldots, s_{v}\right) \mid \forall i \in\right.$ $\left.[1, v]: s_{i} \in \mathcal{M}_{i}\right\}$. With the limitation of possible maneuvers, the integral in (1) is approximated with a sum

$$
P(C) \approx \sum_{s \in \mathcal{S}} P(C \mid s) f(s),
$$

where $f(s)$ is the discrete probability distribution. $P(C \mid s)$ is the risk that a collision occurs in a scene and can be determined with a stochastic collision checker [19] or with a stochastic reachability analysis [13] for all pairs of vehicles in the scene. As each element $s_{i}$ of the tuple $s=$ 
$\left(s_{1}, s_{2}, \ldots, s_{i}, \ldots, s_{v}\right)$ can be assigned a certain maneuver $m_{i, j}$, the probability, that a certain scene $s$ occurs, is

$$
f(s)=\prod_{i=1}^{v} f_{i}\left(s_{i}\right)=\prod_{i=1}^{v} f_{i}\left(m_{i, j}\right)
$$

The probability that vehicle $v_{i}$ performs the maneuver $m_{i, j}$ is denoted by the intention distribution function $f_{i}\left(m_{i, j}\right)$. Preliminary note that (3) assumes stochastic independence of the distributions $f_{i}\left(m_{i, j}\right)$. The value of $f_{i}\left(m_{i, j}\right)$ is defined by a probability distribution of $\mathcal{M}_{i}$. This distribution can be seen as a free space motion including high-level intentions but ignoring other vehicles.

For the computation of the conditional collision risk, depicted as $P\left(C \mid m_{q, p}\right)$, a further set $\mathcal{S}_{q, p} \subseteq \mathcal{S}$ is defined

$$
\mathcal{S}_{q, p}:=\left\{\left(s_{1}, s_{2}, \ldots, s_{q}, \ldots, s_{v}\right) \in \mathcal{S} \mid s_{q}=m_{q, p}\right\}
$$

with $\left|\mathcal{S}_{q, p}\right|=\frac{|\mathcal{S}|}{m_{q}}$, where $\mathcal{S}_{q, p}$ consists of all maneuver combinations $s$ which include the maneuver $m_{q, p}$ of vehicle $v_{q}$. Hence, the conditional collision risk is

$$
P\left(C \mid m_{q, p}\right)=\frac{1}{f_{q}\left(m_{q, p}\right)} \sum_{s \in \mathcal{S}_{q, p}} P(C \mid s) f(s) .
$$

With this information the prior intention estimation is adjusted to obtain an interaction-aware distribution.

\section{Applicability of the Globally Optimal Solution}

If a communication frame was available the overall collision risk of situation $P(C)$ could be cooperatively optimized. But in contrast to [7], this framework covers the case that no centralized cooperation is expected. Instead, it is assumed that the drivers locally optimize their trajectories based on the estimation of the intention of the surrounding drivers.

\section{Implementation}

To improve the prediction of the future movement of the traffic participants the algorithm adapts the prior intention estimations $f_{i}\left(m_{i, j}\right)$ of the maneuvers by computing the collision probabilities $P\left(C \mid m_{i, j}\right)$ and taking the interaction of traffic participants explicitly into account.

1) Calculation of the Collision Probability $P\left(C \mid m_{i, j}\right)$ : Instead of setting up a table of all maneuver permutations, an iterative approach is used, avoiding the need to store the high amount $|\mathcal{S}|$ of all permutations. This approach computes the values $f(s)$ and $P(C \mid s)$ at the same time, when the maneuvers of the vehicles are permuted. After each permutation, the value of the product $f(s) P(C \mid s)$ is added to the corresponding collision probabilities $P\left(C \mid m_{i, j}\right)$ of the maneuvers $m_{i, j}$, which are part of the combination $s$.

The algorithm can be efficiently implemented:

- Initialize $P\left(C \mid m_{i, j}\right)=0$ for all $m_{i, j} \in \mathcal{M}_{i}$ for all $v_{i} \in \mathcal{V}$

- Permute all maneuver combinations $s \in \mathcal{S}$ and add the product $f(s) P(C \mid s)$ of the occurrence probability

$$
f(s)=\prod_{i=1}^{v} f_{i}\left(s_{i}\right)=\prod_{i=1}^{v} f_{i}\left(m_{i, j}\right) .
$$

and the collision risk of the current combination $s$

$$
P(C \mid s)=1-\prod_{i=1}^{v-1} \prod_{k=i+1}^{v}\left(1-P\left(C \mid s_{i}, s_{k}\right)\right) .
$$

to the collision probability $P\left(C \mid m_{i, j}\right)$ for all maneuvers with $m_{i, j}=s_{i}$.

- Divide $P\left(C \mid m_{i, j}\right)$ by $f\left(m_{i, j}\right)$.

With these instructions, the collision probability $P\left(C \mid m_{i, j}\right)$ of each possible maneuver is obtained. Due to the fact that the collision probabilities $P\left(C \mid m_{i, j}\right)$ are now known, it is possible to adapt the prior distribution $f_{i}\left(m_{i, j}\right)$ of each vehicle depending on the value of $P\left(C \mid m_{i, j}\right)$.

\begin{tabular}{|c|c|c|c|c|c|c|c|}
\hline \multirow[b]{2}{*}{$v_{1}$} & \multicolumn{7}{|c|}{ Permutations of the maneuvers } \\
\hline & $m_{1,1}$ & $m_{1,1}$ & $m_{1,1}$ & $m_{1,1}$ & $m_{1,1}$ & $m_{1,1}$ & $\cdots$ \\
\hline$v_{2}$ & $m_{2,1}$ & $m_{2,1}$ & $m_{2,1}$ & $m_{2,2}$ & $m_{2,2}$ & $m_{2,2}$ & $\ldots$ \\
\hline$v_{3}$ & $m_{3,1}$ & $m_{3,2}$ & $m_{3,3}$ & $m_{3,1}$ & $m_{3,2}$ & $m_{3,3}$ & $\ldots$ \\
\hline$f(s)$ & \multicolumn{6}{|c|}{$f_{1}\left(m_{1, \bullet}\right) f_{2}\left(m_{2, \bullet}\right) f_{3}\left(m_{3, \bullet}\right)$} & $\ldots$ \\
\hline$P(C \mid s)$ & \multicolumn{6}{|c|}{$P\left(C \mid m_{1, \bullet}, m_{2, \bullet}, m_{3, \bullet}\right)$} & $\ldots$ \\
\hline
\end{tabular}

An example permutation for the three vehicles in Fig. 4 which are able to execute three different maneuvers is shown in Tab. I. Additionally, the probability $f(s)$ and the collision risk $P(C \mid s)$ of each combination $s$ are given.

TABLE I: Example for the permutation of three vehicles with three possible maneuvers.

2) Modification of the Prior Distributions: How drivers react on their risk estimation should be learned from data. For this interaction model, an exemplary approach distributing the probabilities linearly to the collision risk and the intention estimation is to set $f_{i}^{\text {new }}\left(m_{i, j}\right)=f_{i}\left(m_{i, j}\right) g\left(P\left(C \mid m_{i, j}\right)\right)$ for each maneuver $m_{i, j}$ of each vehicle $v_{i}$ with

$$
g\left(P\left(C \mid m_{i, j}\right)\right)=1-\frac{P\left(C \mid m_{i, j}\right)-\min _{m_{i, k} \in \mathcal{M}_{i}} P\left(C \mid m_{i, k}\right)}{1-\min _{m_{i, k} \in \mathcal{M}_{i}} P\left(C \mid m_{i, k}\right)} .
$$

Of course, $f_{i}^{\text {new }}$ needs to be normalized to one to get $f_{i}^{\text {norm }}\left(m_{i, j}\right)$. In the result $f_{i}^{\text {norm }}\left(m_{i, j}\right)$ the interaction of the drivers has been incorporated explicitly into the intention estimation $f_{i}$ to obtain a more reliable motion prediction. The following shows results of the described algorithm.

\section{RESULTS}

In order to evaluate the presented approach the algorithm was exemplarily implemented in $\mathrm{C}++$. Example simulation road scenes were created and real-world data has been used to test the approach. Therefore, trajectories for the maneuvers were generated and checked for collisions. The presented approach was integrated in the framework of [20].

Minimum jerk trajectories are used to simulate human driving. For computing the trajectories, a road aligned coordinate system is used. As sketched in Fig. 5, this Frenet system consists of two coordinates $s(t)$ and $d(t)$ where the $s$-coordinate runs parallel to the road lane and the $d$ coordinate is perpendicular to the lane. Note that trajectories are represented more realistically in the Frenet frame. A more detailed look at the trajectory generation is given in [21].

As collision checker for the minimum jerk trajectories the approach of [19] is used to obtain the collision risk 


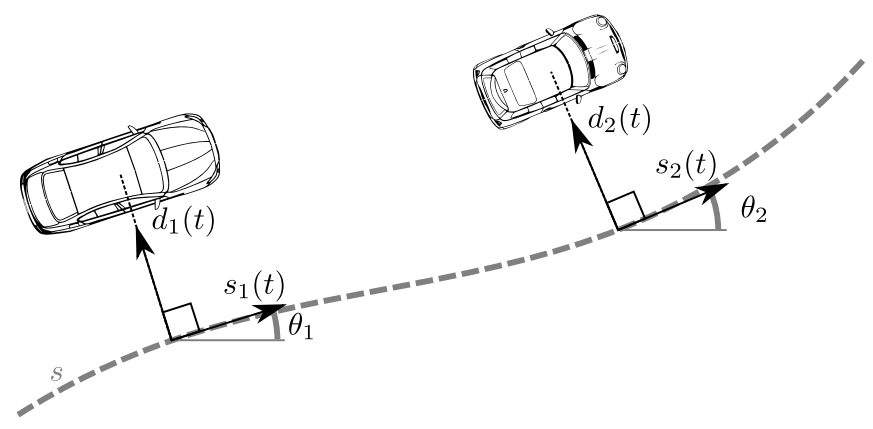

Fig. 5: Illustration of the Frenet frame. The coordinates $s$ and $d$ are the longitudinal and lateral displacement of the center of the street respectively.

$P(C \mid s)$. It tests if a collision will occur between two vehicles when they drive certain maneuvers. For the computation of $P(C \mid s),\left(\begin{array}{l}v \\ 2\end{array}\right)$ possible pairs of maneuvers have to be tested for collision.

Note, that the presented approach is designed to work with arbitrary trajectories and collision checkers.

\section{A. Simulated Highway Scene}

This example serves as a demonstration of the functionality of the presented algorithm. Additionally, the changes from the prior intention estimate to an interaction-aware distribution is shown. With the stochastic collision checker it is possible to compute the values of the collision risks $P\left(C \mid m_{i, j}, m_{k, l}\right), i \neq k$. The possible maneuvers of the vehicles and their prior estimated intention distribution are given in Fig. $6 b$.

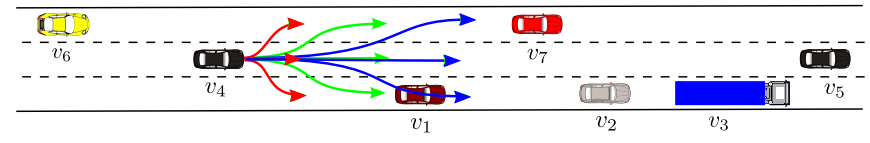

(a) Seven vehicles $v_{1}, \ldots, v_{v}$ on a highway with three lanes. As an example the arrows indicate nine possible maneuvers of vehicle $v_{4}$. To distinguish between the maneuvers brake, keep velocity, and accelerate, the length of the arrows is varied. For clarity, the maneuvers of the others are not drawn.

\begin{tabular}{|c|c|c|c|c|c|c|c|c|c|}
\hline \multirow[t]{3}{*}{$v_{i}$} & \multicolumn{9}{|c|}{ Prior Intention Estimate $f_{i}\left(m_{i, j}\right)$ in $\%$} \\
\hline & \multicolumn{3}{|c|}{ Right lane } & \multicolumn{3}{|c|}{ Middle lane } & \multicolumn{3}{|c|}{ Left lane } \\
\hline & $<0$ & $\approx 0$ & $>0$ & $<0$ & $\approx 0$ & $>0$ & $<0$ & $\approx 0$ & $>0$ \\
\hline$v_{1}$ & 8.0 & 50.0 & 8.0 & 8.0 & 18.0 & 8.0 & - & - & - \\
\hline$v_{2}$ & 8.0 & 50.0 & 8.0 & 8.0 & 18.0 & 8.0 & - & - & - \\
\hline$v_{3}$ & 8.0 & 50.0 & 8.0 & 8.0 & 18.0 & 8.0 & - & - & - \\
\hline$v_{4}$ & 5.0 & 10.0 & 5.0 & 5.0 & 50.0 & 5.0 & 5.0 & 10.0 & 5.0 \\
\hline$v_{5}$ & 5.0 & 10.0 & 5.0 & 5.0 & 50.0 & 5.0 & 5.0 & 10.0 & 5.0 \\
\hline$v_{6}$ & - & - & - & 8.0 & 18.0 & 8.0 & 8.0 & 50.0 & 8.0 \\
\hline$v_{7}$ & - & - & - & 8.0 & 18.0 & 8.0 & 8.0 & 50.0 & 8.0 \\
\hline
\end{tabular}

(b) This table lists the probabilities, that drivers perform a certain maneuver. These values have yet not been modified.

\begin{tabular}{cccccccc}
\hline$v_{i}$ & $v_{1}$ & $v_{2}$ & $v_{3}$ & $v_{4}$ & $v_{5}$ & $v_{6}$ & $v_{7}$ \\
\hline Speed in $\mathrm{km} / \mathrm{h}$ & 140 & 100 & 80 & 160 & 110 & 200 & 180 \\
\hline
\end{tabular}

(c) The vehicles drive at different initial speeds which are listed in this table.

Fig. 6: Simulation highway scenario.

All vehicles are assumed to be able to brake $(a<0)$, accelerate $(a>0)$ or to keep their velocity $(a=0)$ with $a$ as longitudinal acceleration. In addition they have the opportunity to stay on their lane or to change to adjacent lanes. Lane changes over two lanes are not allowed. In future studies, the chosen discretization has to be verified with real world data. For example, the probability that vehicle $v_{1}$ keeps its lane and velocity, is assumed to be $50 \%$. The vehicles $v_{4}$ and $v_{5}$ are on the middle lane as sketched in Fig. 6a. The figure contains a highway with three lanes and seven vehicles $v_{i}$, which are moving with different speeds. Their speeds are listed in Fig. 6c.

Applying the stochastic collision checker to this highway scenario, the values of Fig. 7a are obtained for the collision probabilities $P\left(C \mid m_{i, j}\right)$. Note how high and unrealistic these values are as no interaction has been integrated yet.

\begin{tabular}{|c|c|c|c|c|c|c|c|c|c|}
\hline \multirow[t]{3}{*}{$v_{i}$} & \multicolumn{9}{|c|}{ Collision Probabilities $P\left(C \mid m_{i, j}\right)$ in $\%$} \\
\hline & \multicolumn{3}{|c|}{ Right lane } & \multicolumn{3}{|c|}{ Middle lane } & \multicolumn{3}{|c|}{ Left lane } \\
\hline & $<0$ & $\approx 0$ & $>0$ & $<0$ & $\approx 0$ & $>0$ & $<0$ & $\approx 0$ & $>0$ \\
\hline$v_{1}$ & 86.0 & 85.9 & 98.6 & 94.4 & 86.4 & 94.6 & - & - & - \\
\hline$v_{2}$ & 90.9 & 90.6 & 93.6 & 88.6 & 81.8 & 81.9 & - & - & - \\
\hline$v_{3}$ & 97.4 & 88.8 & 79.5 & 96.0 & 86.0 & 83.4 & - & - & - \\
\hline$v_{4}$ & 82.8 & 85.0 & 85.6 & 88.1 & 88.3 & 88.9 & 94.7 & 91.5 & 91.5 \\
\hline$v_{5}$ & 98.2 & 71.6 & 71.6 & 98.5 & 90.1 & 90.1 & 80.0 & 95.2 & 95.2 \\
\hline$v_{6}$ & - & - & - & 76.5 & 84.1 & 88.0 & 75.6 & 93.2 & 93.2 \\
\hline$v_{7}$ & - & - & - & 97.5 & 92.9 & 92.9 & 85.5 & 85.5 & 85.5 \\
\hline
\end{tabular}

(a) The collision probabilities $P\left(C \mid m_{i, j}\right)$ of vehicles, performing certain maneuvers, are listed.

\begin{tabular}{|c|c|c|c|c|c|c|c|c|c|}
\hline \multirow[t]{3}{*}{$v_{i}$} & \multicolumn{9}{|c|}{ Interaction-aware Distribution $f_{i}^{\text {norm }}\left(m_{i, j}\right)$ in $\%$} \\
\hline & \multicolumn{3}{|c|}{ Right lane } & \multicolumn{3}{|c|}{ Middle lane } & \multicolumn{3}{|c|}{ Left lane } \\
\hline & $<0$ & $\approx 0$ & $>0$ & $<0$ & $\approx 0$ & $>0$ & $<0$ & $\approx 0$ & $>0$ \\
\hline$v_{1}$ & 9.7 & 60.7 & 0.9 & 3.9 & 21.1 & 3.7 & - & 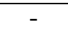 & - \\
\hline$v_{2}$ & 6.3 & 40.6 & 4.4 & 7.9 & 28.3 & 12.5 & - & - & - \\
\hline$v_{3}$ & 1.8 & 48.2 & 14.1 & 2.8 & 21.7 & 11.4 & - & - & - \\
\hline$v_{4}$ & 7.4 & 12.9 & 6.2 & 5.1 & 50.3 & 4.8 & 2.3 & 7.3 & 3.7 \\
\hline$v_{5}$ & 0.8 & 24.5 & 12.3 & 0.7 & 42.7 & 4.3 & 8.6 & 4.1 & 2.1 \\
\hline$v_{6}$ & - & - & - & 16.2 & 24.7 & 8.3 & 16.8 & 29.3 & 4.7 \\
\hline$v_{7}$ & - & - & - & 1.7 & 11.0 & 4.9 & 10.0 & 62.2 & 10.3 \\
\hline
\end{tabular}

(b) The interaction-aware maneuver probabilities are estimated from the intention estimation and the collision probabilities.

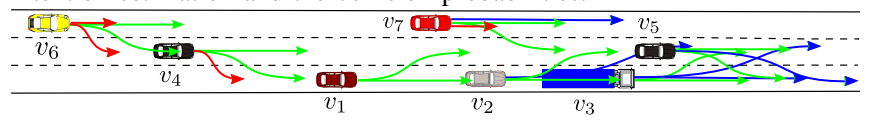

(c) The figure sketches all maneuvers of the interaction-aware prediction with the probability $f_{i}^{\text {norm }}\left(m_{i, j}\right)>10 \%$ with arrows. Red arrows indicate braking maneuvers, while green arrows and blue arrows illustrate maintaining the current speed and longitudinal acceleration respectively.

Fig. 7: Results of the collision checker and the presented algorithm.

In order to modify the prior distribution, the adjustment step of IV-D.2 is applied to the collision probabilities. The results are presented in Fig. $7 \mathrm{~b}$.

This scenario has been computed on an off-the-shelf Intel Core i7-2600 with $3.4 \mathrm{GHz}$ in a multi-threaded $\mathrm{C}++$ exemplary implementation. The $|\mathcal{S}|=629,856$ scenarios of this example have been calculated in less than $4.5 \mathrm{~ms}$, so the computational time is very promising even for more complex scenarios. In Fig. 7c the most probable future actions $f_{i}^{\text {norm }}\left(m_{i, j}\right)>10 \%$ of the vehicles are illustrated by the arrows of the vehicles.

\section{B. Real-world Highway Scene}

The presented algorithm has also been tested with recorded data from a German highway scene. Fig. 8 shows a frame of the trip that is evaluated with the novel approach. Again, the vehicles have been assigned an intention estimation analog the values in Fig. 6b. In future implementations these values 


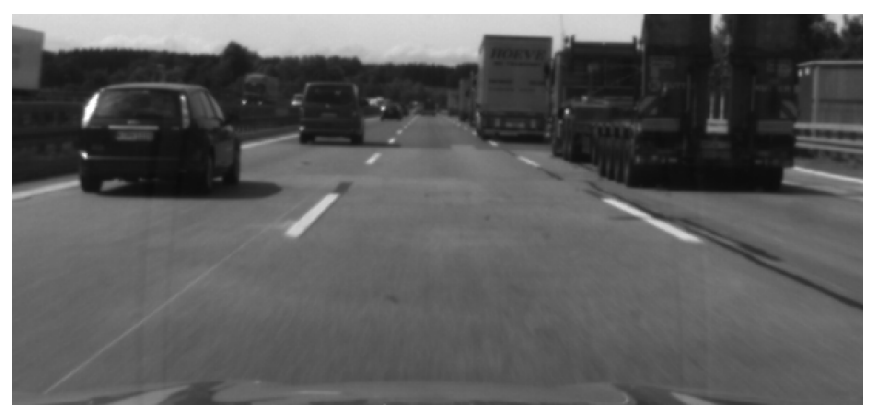

(a) Photo taken with the front camera on a highway scene with several surrounding vehicles.

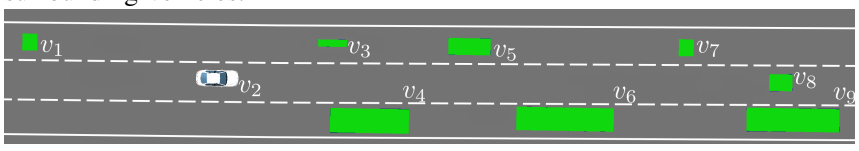

(b) With sensor data and tracking system constructed top view of this scene. The ego vehicle is painted in white, all surrounding vehicles in green.

Fig. 8: Real-world scene with multiple vehicles from a trip on a highway.

are chosen according to a cost function that penalizes jerks, deviations e.g. from the lane and time.

In Fig. 9 the results of the algorithm are presented. The situation with $|\mathcal{S}|=22,674,816$ has been predicted in $342.5 \mathrm{~ms}$ on the same processor.

\begin{tabular}{|c|c|c|c|c|c|c|c|c|c|}
\hline \multirow[t]{3}{*}{$v_{i}$} & \multicolumn{9}{|c|}{ Interaction-aware Distribution $f_{i}^{\text {norm }}\left(m_{i, j}\right)$ in $\%$} \\
\hline & \multicolumn{3}{|c|}{ Right lane } & \multicolumn{3}{|c|}{ Middle lane } & \multicolumn{3}{|c|}{ Left lane } \\
\hline & $<0$ & $\approx 0$ & $>0$ & $<0$ & $\approx 0$ & $>0$ & $<0$ & $\approx 0$ & $>0$ \\
\hline$v_{1}$ & - & - & 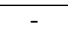 & 10.0 & 5.8 & 2.6 & 10.0 & 62.5 & 9.2 \\
\hline$v_{2}$ & 0.0 & 0.0 & 0.0 & 6.2 & 61.8 & 8.4 & 11.4 & 8.1 & 4.1 \\
\hline$v_{3}$ & - & - & - & 9.5 & 20.2 & 9.4 & 7.0 & 43.8 & 10.1 \\
\hline$v_{4}$ & 13.6 & 77.0 & 5.0 & 1.5 & 2.2 & 0.8 & - & - & - \\
\hline$v_{5}$ & - & - & - & 4.1 & 13.2 & 6.9 & 4.5 & 62.4 & 8.9 \\
\hline$v_{6}$ & 1.8 & 56.0 & 10.1 & 8.3 & 16.5 & 7.4 & - & - & - \\
\hline$v_{7}$ & - & - & - & 5.7 & 10.9 & 4.9 & 9.7 & 59.4 & 9.5 \\
\hline$v_{8}$ & 0.0 & 0.0 & 0.0 & 7.3 & 64.6 & 6.5 & 5.2 & 10.3 & 6.3 \\
\hline$v_{9}$ & 3.9 & 76.4 & 12.2 & 1.5 & 4.2 & 1.9 & - & - & - \\
\hline
\end{tabular}

Fig. 9: First results for a real-world scenario on a highway: The posterior probabilities, that drivers perform a certain maneuver respecting the current environment, are given in this table.

\section{CONCLUSIONS}

In this paper, a novel approach for motion prediction and threat assessment of objects in structured environments, like highways or crossings, is presented. The approach is developed for driver assistance systems as well as for autonomous driving. The systems assess the danger of possible future trajectories. For that purpose, they require a reliable prediction of the movements of other traffic participants. Due to the fact that the drivers have a mutual influence on each other, it is necessary to consider the interaction of the traffic participants in order to obtain a reliable prediction. Taking the interaction explicitly into account, this framework offers reliability and differs from already existing approaches which do not consider the interaction sufficiently. In simulated and real-world scenarios, the computation time of an exemplary implementation of this approach is shown to meet the on-line requirements. Future work includes evaluation of the chosen interaction model, tests with inter-vehicle communication and different environment settings.

\section{ACKNOWLEDGMENT}

The authors gratefully acknowledge support of this work by the BMW Group Research and Technology, Munich, Germany. In part this work is supported by the DFG excellence initiative research cluster Cognition for Technical Systems (CoTeSys), by the Institute for Advanced Study (IAS), Technische Universität München, Munich, Germany and by the ERC Advanced Grant SHRINE.

\section{REFERENCES}

[1] U.S. Dept, of Transportation - National Hightway Traffic Safety Administration, "Fatatlity analysis reporting system," 2009.

[2] C. Hermes, C. Wohler, K. Schenk, and F. Kummert, "Long-term vehicle motion prediction," in IEEE Intelligent Vehicles Symp., 2009, pp. 652-657.

[3] D. Vasquez, F. Large, T. Fraichard, and C. Laugier, "High-speed autonomous navigation with motion prediction for unknown moving obstacles," in IEEE/RSJ Int. Conf. on Intelligent Robots and Systems, vol. 1, 2004, pp. 82-87.

[4] D. Vasquez and T. Fraichard, "Motion prediction for moving objects: a statistical approach," in IEEE Int. Conf. on Robotics and Automation, vol. 4, 2004, pp. 3931-3936.

[5] D. Vasquez, T. Fraichard, and C. Laugier, "Incremental learning of statistical motion patterns with growing hidden markov models," IEEE Transactions on Intelligent Transportation Systems, vol. 10, no. 3, pp. 403-416, 2009.

[6] D. Vasquez, Incremental Learning for Motion Prediction of Pedestrians and Vehicles, ser. Springer Tracts in Advanced Robotics. Springer, 2010, vol. 64.

[7] C. Frese, Planung kooperativer Fahrmanöver für kognitive Automobile, ser. Karlsruher Schriften zur Anthropomatik. KIT Scientific Publishing, 2012, vol. 10.

[8] D. D. Salvucci, "Modeling driver behavior in a cognitive architecture," Human Factors: The Journal of the Human Factors and Ergonomics Society, vol. 48, no. 2, pp. 362-380, 2006.

[9] A. Polychronopoulos, M. Tsogas, A. Amditis, U. Scheunert, L. Andreone, and F. Tango, "Dynamic situation and threat assessment for collision warning systems: the euclide approach," in IEEE Intelligent Vehicles Symp., 2004, pp. 636-641.

[10] J. Gunnarsson, L. Svensson, E. Bengtsson, and L. Danielsson, "Joint driver intention classification and tracking of vehicles," in IEEE Nonlinear Statistical Signal Processing Workshop, 2006, pp. 95-98.

[11] J. Sorstedt, L. Svensson, F. Sandblom, and L. Hammarstrand, "A new vehicle motion model for improved predictions and situation assessment," IEEE Transactions on Intelligent Transportation Systems, vol. 12, no. 4, pp. 1209-1219, 2011.

[12] M. Tsogas, A. Polychronopoulos, N. Floudas, and A. Amditis, "Situation refinement for vehicle maneuver identification and driver's intention prediction," in Int. Conf. on Information Fusion, 2007, pp. 1-8.

[13] M. Althoff, O. Stursberg, and M. Buss, "Safety assessment of driving behavior in multi-lane traffic for autonomous vehicles," in IEEE Intelligent Vehicles Symp., 2009, pp. 893-900.

[14] A. Broadhurst, S. Baker, and T. Kanade, "Monte carlo road safety reasoning," in IEEE Intelligent Vehicles Symp., 2005, pp. 319-324.

[15] A. Eidehall and L. Petersson, "Statistical threat assessment for general road scenes using monte carlo sampling," IEEE Transactions on Intelligent Transportation Systems, vol. 9, no. 1, pp. 137-147, 2008.

[16] S. Lefèvre, C. Laugier, and J. Ibañez-Guzmán, "Evaluating risk at road intersections by detecting conflicting intentions," in IEEE/RSJ Int. Conf. on Intelligent Robots and Systems, 2012, pp. 4841-4846.

[17] — "Risk assessment at road intersections: Comparing intention and expectation," in IEEE Intelligent Vehicles Symp., 2012, pp. 165-171.

[18] J. van den Berg, S. J. Guy, M. C. Lin, and D. Manocha, "Reciprocal n-body collision avoidance," in Robotics Research, 2011, pp. 3-19.

[19] A. Lawitzky, D. Wollherr, and M. Buss, "Maneuver-based risk assessment for high-speed automotive scenarios," in IEEE/RSJ Int. Conf. on Intelligent Robots and Systems, 2012, pp. 1186-1191.

[20] D. Althoff, M. Buss, A. Lawitzky, M. Werling, and D. Wollherr, "On-line trajectory generation for safe and optimal vehicle motion planning," in Autonome Mobile Systeme. Springer, 2012.

[21] M. Werling, S. Kammel, J. Ziegler, and L. Groell, "Optimal trajectories for time-critical street scenarios using discretized terminal manifolds," International Journal of Robotics Research, pp. 346-359, 2012. 